EPJ Web of Conferences 108, 02034 (2016)

DOI: $10.1051 /$ epjconf/201610802034

(C) Owned by the authors, published by EDP Sciences, 2016

\title{
Spinor-Like Hamiltonian for Maxwellian Optics
}

\author{
D. S. Kulyabovi,2,a \\ ${ }^{1}$ Department of Applied Probability and Informatics, Peoples' Friendship University of Russia, \\ Miklukho-Maklaya str. 6, Moscow, 117198, Russia \\ ${ }^{2}$ Laboratory of Information Technologies, Joint Institute for Nuclear Research, \\ Joliot-Curie 6, Dubna, Moscow region, 141980, Russia
}

\begin{abstract}
Background. Spinors are more special objects than tensors. Therefore spinors possess more properties than the more generic objects such as tensors. The group of Lorentz two-spinors is the covering group of the Lorentz group.

Purpose. Since the Lorentz group is the symmetry group of Maxwell equations, it is reasonable to use Lorentz two-spinors and not tensors when writing the Maxwell equations. MeтноD. We write the Maxwell equations using Lorentz two-spinors. Also a convenient representation of Lorentz two-spinors in terms of the Riemann-Silberstein complex vectors is used.

Results. In the spinor formalism (in the representation of the Lorentz spinors and Riemann-Silberstein vectors) we have constructed the Hamiltonian of Maxwellian optics. With the use of spinors, the Maxwell equations take a form similar to the Dirac equation.

Conclusions. For Maxwell equations in the Dirac-like form we can expand research methods by means of quantum field theory. In this form, the connection between the Hamiltonians of geometric, beam and Maxwellian optics is clearly visible.
\end{abstract}

\section{Introduction}

The Maxwell equations have a large number of representations [1]. The principle of this study is the following: every representation must simplify the concrete theoretical and practical study. The geometrization of Maxwell equations and the Hamiltonian formalism are of interest.

In this paper, on the basis of spinor representation [2-5] of Maxwell equations, we propose to construct the Dirac-like Hamiltonian. It is expected that this form will allow us to apply the quantum formalism to the study of Maxwell equations.

The structure of the article is as follows. In the section 2 the basic notations and conventions are introduced. Section 3 gives a brief description of the Maxwell equations. Section 4 introduces the complex representation of the Maxwell equations. Section 5 gives the spinor representation of the Maxwell equations. In section 6, using a combination of the results of the two previous sections, we obtain a Dirac-like Hamiltonian of Maxwell equations.

a e-mail: yamadharma@gmail.com 


\section{Notations and conventions}

1. The abstract indices notation [6] is used. Under this notation a tensor as a whole object is denoted just as an index (e.g., $x^{i}$ ), components are denoted by an underlined index (e.g., $x_{-}^{i}$ ).

2. We will adhere to the following conventions. Greek indices $(\alpha, \beta)$ will refer to the fourdimensional space, in component form it looks like: $\alpha=\overline{0,3}$. Latin indices from the middle of the alphabet $(i, j, k)$ will refer to the three-dimensional space, in the component form it looks like: $i=\overline{1,3}$.

3. The comma in the index denotes partial derivative with respect to the corresponding coordinate $\left(f_{, i}:=\partial_{i} f\right)$; a semicolon denotes the covariant derivative $\left(f_{; i}:=\nabla_{i} f\right)$.

4. The equations of the electrodynamics are written in the CGS symmetrical system.

5. Antisymmetrization is denoted by straight brackets.

\section{Maxwell Equations}

The Maxwell equations in 3-dimensional form:

$$
\begin{gathered}
\nabla_{0} B^{i}=-e^{i j k} \nabla_{j} E_{k} ; \\
\nabla_{i} D^{i}=4 \pi \rho ; \\
\nabla_{0} D^{i}=e^{i j k} \nabla_{j} H_{k}-\frac{4 \pi}{c} j^{i} ; \\
\nabla_{i} B^{i}=0 .
\end{gathered}
$$

where $e^{i j k}$ is the alternating tensor expressed by the Levi-Civita simbol $\varepsilon^{i j k}$ :

$$
e_{\underline{i j k}}=\sqrt{{ }^{3} g} \varepsilon_{\underline{i j k}}, \quad e \underline{i j k}=\frac{1}{\sqrt{{ }^{3} g}} \varepsilon^{i j k} .
$$

With the help of the electromagnetic field tensors $F_{\alpha \beta}$ and $G_{\alpha \beta}$ [7], the system (1) becomes:

$$
\begin{gathered}
\nabla_{\alpha} F_{\beta \gamma}+\nabla_{\beta} F_{\gamma \alpha}+\nabla_{\gamma} F_{\alpha \beta}=F_{[\alpha \beta ; \gamma]}=0, \\
\nabla_{\alpha} G^{\alpha \beta}=\frac{4 \pi}{c} j^{\beta}
\end{gathered}
$$

where

$$
F_{\underline{\alpha \beta}}=\left(\begin{array}{cccc}
0 & E_{1} & E_{2} & E_{3} \\
-E_{1} & 0 & -B^{3} & B^{2} \\
-E_{2} & B^{3} & 0 & -B^{1} \\
-E_{3} & -B^{2} & B^{1} & 0
\end{array}\right), \quad G \underline{\alpha \beta}=\left(\begin{array}{cccc}
0 & -D^{1} & -D^{2} & -D^{3} \\
D^{1} & 0 & -H_{3} & H_{2} \\
D^{2} & H_{3} & 0 & -H_{1} \\
D^{3} & -H_{2} & H_{1} & 0
\end{array}\right),
$$

$E_{i}, H_{-}^{i}, i=\overline{1,3}$, denote the components of the electric and the magnetic fields intensity vectors; $D_{i}$, $B_{-}^{\bar{i}}, i=\overline{1,3}$, the components of the electric and magnetic induction vectors.

\section{Complex Form of Maxwell Equations}

The complex form of the Maxwell equations was considered by various authors [8-10]. 


\subsection{Generic representation}

The following correspondences between ordered pairs and complex 3-vectors are defined,

$$
\begin{aligned}
F_{-}^{i} \sim\left(E_{-}^{i}, B_{-}^{i}\right), & F_{-}^{i}=E_{-}^{i}+\mathrm{i} B_{-}^{i} ; \\
G_{-}^{i} \sim\left(D_{-}^{i}, H_{-}^{i}\right), & G_{-}^{i}=D_{-}^{i}+\mathrm{i} H_{-}^{i} .
\end{aligned}
$$

Then the intensity and the induction can be expressed in terms of complex vectors,

$$
\begin{array}{ll}
E^{i}=\frac{F^{i}+\bar{F}^{i}}{2}, & B^{i}=\frac{F^{i}-\bar{F}^{i}}{2 \mathrm{i}}, \\
D^{i}=\frac{G^{i}+\bar{G}^{i}}{2}, & H^{i}=\frac{G^{i}-\bar{G}^{i}}{2 \mathrm{i}} .
\end{array}
$$

Using the two complementary vectors,

$$
K^{i}=\frac{G^{i}+F^{i}}{2}, \quad L^{i}=\frac{\bar{G}^{i}-\bar{F}^{i}}{2} .
$$

the expression (1) assumes the form

$$
\begin{gathered}
\nabla_{i}\left(K^{i}+L^{i}\right)=4 \pi \rho ; \\
-\mathrm{i} \nabla_{0}\left(K^{i}-L^{i}\right)+e^{i j k} \nabla_{j}\left(K_{k}-L_{k}\right)=\mathrm{i} \frac{4 \pi}{c} j^{i} .
\end{gathered}
$$

\subsection{Complex Form of Maxwell Equations in Vacuum}

From $D^{i}=E^{i}, H^{i}=B^{i}$ and (3) it follows

$$
K^{i}=E^{i}+\mathrm{i} B^{i}=F^{i}, \quad L^{i}=0 .
$$

Then the equations (4) will have the form

$$
\begin{gathered}
\nabla_{i} F^{i}=4 \pi \rho ; \\
-\mathrm{i} \nabla_{0} F^{i}+e^{i j k} \nabla_{j} F_{k}=\mathrm{i} \frac{4 \pi}{c} j^{i} .
\end{gathered}
$$

\subsection{Complex Representation of Maxwell Equations in Homogeneous Isotropic Space}

In the homogeneous isotropic space, the relationships $D^{i}=\varepsilon E^{i}, \mu H^{i}=B^{i}$ (where $\varepsilon$ denotes the dielectric permittivity and $\mu$, the magnetic permeability) hold.

The resulting expressions may be simplified as follows. In (5) we need the formal substitutions $c \rightarrow c^{\prime}=\frac{c}{\sqrt{\varepsilon \mu}}$ (the speed of light in vacuum is substituted by the speed of light in medium) and $j^{\alpha} \rightarrow \frac{j^{\alpha}}{\sqrt{\varepsilon}}$. The result is:

$$
F^{i}=\sqrt{\varepsilon} E^{i}+\mathrm{i} \frac{1}{\sqrt{\mu}} B^{i} .
$$

Then the Maxwell equations (1) take the form:

$$
\begin{gathered}
\nabla_{i} F^{i}=\frac{4 \pi}{\sqrt{\varepsilon}} \rho ; \\
\mathrm{i} \frac{1}{c} \frac{\partial F^{i}}{\partial t}=\frac{1}{\sqrt{\varepsilon \mu}} e^{i j k} \nabla_{j} F_{k}-\mathrm{i} \frac{4 \pi}{c \sqrt{\varepsilon}} j^{i} .
\end{gathered}
$$


This representation of Maxwell equations has several names. In particular, it is known as the Riemann-Silberstein representation [8,9].

\section{Spinor Form of Maxwell Equations}

The electromagnetic field tensor $F_{\alpha \beta}$ and its components $F_{\underline{\alpha \beta}}, \underline{\alpha}, \underline{\beta}=\overline{0,3}$ may be considered in spinor form [6] (and similarly for $G_{\alpha \beta}$ ):

$$
\begin{aligned}
& F_{\alpha \beta}=F_{A A^{\prime} B B^{\prime}} ; \\
& F_{\underline{\alpha} \underline{\beta}}=F_{\underline{A} \underline{A}^{\prime} \underline{B} \underline{B}^{\prime}} g_{\underline{\alpha}}{ }^{-}{ }^{-A^{\prime}} g_{\underline{\beta}^{-}}{ }^{B} B^{\prime}, \\
& \underline{A}, A^{\prime}, \underline{B}, \underline{B}^{\prime}=\overline{0,1}, \quad \underline{\alpha}, \underline{\beta}=\overline{0,3},
\end{aligned}
$$

where $g_{\underline{\alpha}}{ }^{A} A^{\prime}$ denote the Infeld-van der Waerden symbols defined in the real spinor basis $\varepsilon_{-\underline{B}}$ as [6]:

$$
\begin{aligned}
& g_{\underline{\alpha}}{ }^{A A^{\prime}}:=g_{\underline{\alpha}}{ }^{\alpha} \varepsilon_{A}{ }^{A} \varepsilon_{A^{\prime}}{ }^{A^{\prime}}, \quad g_{\underline{A} A^{\prime}} \underline{\alpha}:=g_{-\alpha}^{\alpha} \varepsilon^{A}{ }_{A} \varepsilon^{A^{A^{\prime}}}{ }_{A^{\prime}}, \\
& \varepsilon_{\underline{A} \underline{B}}=\varepsilon_{\underline{A^{\prime}} \underline{B}^{\prime}}=\left(\begin{array}{cc}
0 & 1 \\
-1 & 0
\end{array}\right), \quad \varepsilon_{\underline{A}}{ }^{A} \varepsilon_{A^{-}}{ }^{\underline{B}}=\varepsilon_{\underline{A}}{ }^{\underline{B}}=\left(\begin{array}{ll}
1 & 0 \\
0 & 1
\end{array}\right) .
\end{aligned}
$$

The Maxwell equations are reformulated in what follows, using the spinor notations.

The tensor $F_{\alpha \beta}$ is real and antisymmetric, it can be represented in the form

$$
\begin{gathered}
F_{\alpha \beta}=\varphi_{A B} \varepsilon_{A^{\prime} B^{\prime}}+\varepsilon_{A B} \bar{\varphi}_{A^{\prime} B^{\prime}}, \\
{ }^{*} F^{\alpha \beta}=-\mathrm{i} \varphi^{A B} \varepsilon^{A^{\prime} B^{\prime}}+\mathrm{i} \varepsilon^{A B} \bar{\varphi}^{A^{\prime} B^{\prime}} .
\end{gathered}
$$

where $\varphi_{A B}$ is the spinor of the electromagnetic field:

$$
\varphi_{A B}:=\frac{1}{2} F_{A B C^{\prime}} C^{\prime}=\frac{1}{2} F_{A A^{\prime} B B^{\prime}} \varepsilon^{A^{\prime} B^{\prime}}=\frac{1}{2} F_{\alpha \beta} \varepsilon^{A^{\prime} B^{\prime}} .
$$

Similarly

$$
\begin{gathered}
G^{\alpha \beta}=\gamma^{A B} \varepsilon^{A^{\prime} B^{\prime}}+\varepsilon^{A B} \bar{\gamma}^{A^{\prime} B^{\prime}}, \\
{ }^{*} G_{\alpha \beta}=-\mathrm{i} \gamma_{A B} \varepsilon_{A^{\prime} B^{\prime}}+\mathrm{i} \varepsilon_{A B} \bar{\gamma}_{A^{\prime} B^{\prime}} .
\end{gathered}
$$

Replacing in (2) the abstract indices $\alpha$ by $A A^{\prime}$ and $\beta$ by $B B^{\prime}$, we can write:

$$
\nabla_{A A^{\prime}} G^{A A^{\prime} B B^{\prime}}=\frac{4 \pi}{c} j^{B B^{\prime}} .
$$

Using (12) we will get

$$
\nabla^{A B^{\prime}} \gamma_{A}^{B}+\nabla^{B A^{\prime}} \gamma_{A^{\prime}}^{B^{\prime}}=\frac{4 \pi}{c} j^{B B^{\prime}}
$$

Similarly, from (10) it follows

$$
\nabla^{A^{\prime} B} \varphi_{B}^{A}-\nabla^{A B^{\prime}} \bar{\varphi}_{B^{\prime}}^{A^{\prime}}=0
$$

As a result, the system of the Maxwell equations can be written as

$$
\begin{gathered}
\nabla^{A^{\prime} B} \varphi_{B}^{A}-\nabla^{A B^{\prime}} \bar{\varphi}_{B^{\prime}}^{A^{\prime}}=0, \\
\nabla^{A B^{\prime}} \gamma_{A}^{B}+\nabla^{B A^{\prime}} \gamma_{A^{\prime}}^{B^{\prime}}=\frac{4 \pi}{c} j^{B B^{\prime}} .
\end{gathered}
$$


In spinor form, the system of Maxwell equations in vacuum can be written as a single equation [6]:

$$
\nabla^{A B^{\prime}} \varphi_{A}^{B}=\frac{2 \pi}{c} j^{B B^{\prime}}
$$

The components of electromagnetic field spinor are:

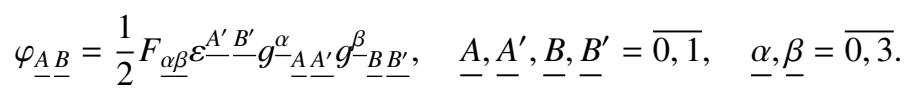

Using the equations (8), (9) and the notation $F_{i}=E_{i}-\mathrm{i} B^{i}$, we get:

$$
\begin{gathered}
\varphi_{00}=\frac{1}{2}\left(F_{1}-\mathrm{i} F_{2}\right), \\
\varphi_{01}=\varphi_{10}=-\frac{1}{2} F_{3}, \\
\varphi_{11}=-\frac{1}{2}\left(F_{1}+\mathrm{i} F_{2}\right) .
\end{gathered}
$$

\section{Dirac-like Hamiltonian}

Using (6)), the spinor of valence one $\psi^{a}$ is constructed from the spinor of valence two $\varphi_{A B}(11)$ as:

$$
\psi^{a}=\left(\begin{array}{l}
\psi^{1} \\
\psi^{2} \\
\psi^{3} \\
\psi^{4}
\end{array}\right)=2\left(\begin{array}{c}
\varphi^{00} \\
\varphi^{01} \\
\varphi^{10} \\
\varphi^{11}
\end{array}\right)=\left(\begin{array}{c}
-F^{1}+\mathrm{i} F^{2} \\
F^{3} \\
F^{3} \\
F^{1}+\mathrm{i} F^{2}
\end{array}\right)
$$

The current takes the form:

$$
\xi^{a}=\left(\begin{array}{c}
-j^{1}+\mathrm{i} j^{2} \\
j^{3}-c \rho \\
j^{3}+c \rho \\
j^{1}+\mathrm{i} j^{2}
\end{array}\right)
$$

Then the system of equations (7) takes the following Dirac-like form:

$$
\frac{1}{c} \frac{\partial \psi^{a}}{\partial t}=-\frac{1}{\sqrt{\varepsilon \mu}} e^{i j k} \nabla_{j} \gamma_{k b}^{a} \psi^{b}-\frac{4 \pi}{c \sqrt{\varepsilon}} \xi^{a}
$$

where the Infeld-van der Waerden symbols $\gamma_{\underline{k}} \underset{\underline{k}}{\underline{b}}$ can be represented as:

$$
\gamma_{1 \underset{-}{b}}^{\stackrel{a}{b}}=\left(\begin{array}{cccc}
0 & 0 & 1 & 0 \\
0 & 0 & 0 & 1 \\
1 & 0 & 0 & 0 \\
0 & 1 & 0 & 0
\end{array}\right), \quad \gamma_{2}^{\underset{b}{b}}=\left(\begin{array}{cccc}
0 & 0 & -\mathrm{i} & 0 \\
0 & 0 & 0 & -\mathrm{i} \\
\mathrm{i} & 0 & 0 & 0 \\
0 & \mathrm{i} & 0 & 0
\end{array}\right), \quad \gamma_{3}^{\underline{a}} \underset{-}{\underline{b}}=\left(\begin{array}{cccc}
1 & 0 & 0 & 0 \\
0 & 1 & 0 & 0 \\
0 & 0 & -1 & 0 \\
0 & 0 & 0 & -1
\end{array}\right) \text {. }
$$

From the structure of the Dirac equation

$$
\mathrm{i} \hbar \frac{\mathrm{d} \psi}{\mathrm{d} t}=\left[c \gamma^{i} p_{i}+\gamma^{0} m c^{2}\right] \psi=H \psi
$$

we get the Dirac-like Hamiltonian of the Maxwell equations with no currents,

$$
H=-\frac{c}{\sqrt{\varepsilon \mu}} e^{i j k} \nabla_{j} \gamma_{k b}^{a} \text {. }
$$




\section{Conclusions}

A method for obtaining the Dirac-like Hamiltonian of the Maxwell equations was proposed. This might allow the application of quantum theory methods to the study of electromagnetic phenomena.

\section{Acknowledgements}

The work is partially supported by RFBR grants No's 14-01-00628 and 15-07-08795.

\section{References}

[1] D.S. Kulyabov, A.V. Korolkova, V.I. Korolkov, Bulletin of Peoples' Friendship University of Russia. Series "Mathematics. Information Sciences. Physics" pp. 96-106 (2012), [arXiv]1211.6590

[2] O. Laporte, G.E. Uhlenbeck, Physical Review 37, 1380 (1931)

[3] I. Bialynicki-Birula, in Progress in Optics (Elsevier, 1996), Vol. 36, chap. 5, pp. 245-294, [arXiv]0508202

[4] S.A. Khan, Physica Scripta 71, 440 (2005), [arXiv]physics/0205083v1

[5] A. Aste, Journal of Geometry and Symmetry in Physics pp. 47-58 (2012), [arXiv]1211.1218v3

[6] R. Penrose, W. Rindler, Spinors and Space-Time: Volume 1, Two-Spinor Calculus and Relativistic Fields, Vol. 1 (Cambridge University Press, 1987), ISBN 0521337070

[7] H. Minkowski, Math. Ann. pp. 472-525 (1910)

[8] L. Silberstein, Annalen der Physik 327, 579 (1907)

[9] L. Silberstein, Annalen der Physik 329, 783 (1907)

[10] J.A. Stratton, Electromagnetic Theory (MGH, 1941) 